\title{
Correction to: Optimizing Pueraria candollei var. mirifica cell suspension culture for prolonged maintenance and decreased variation of isoflavonoid from single cell lines
}

\author{
Dolly Rani ${ }^{1} \cdot$ Thitirat Meelaph $^{2} \cdot$ Khwanlada Kobtrakul $^{2} \cdot$ Sornkanok Vimolmangkang ${ }^{1,3}$ (1)
}

Published online: 3 January 2020

(c) Springer Nature B.V. 2020

\section{Correction to: \\ Plant Cell, Tissue and Organ Culture (PCTOC) \\ (2018) 134:433-443 \\ https://doi.org/10.1007/s11240-018-1433-6}

The Acknowledgements section in the original article was incomplete. The complete section is shown below.

Acknowledgements This research was supported by Rachadapisek Sompote Fund for Postdoctoral Fellowship and for New Faculty Member Chulalongkorn University, Bangkok and Thailand Research
Fund Project No. MRG5680090. TM was a recipient of the Research assistant scholarship from the Graduate School, Chulalongkorn University.

Publisher's Note Springer Nature remains neutral with regard to jurisdictional claims in published maps and institutional affiliations.

The original article can be found online at https://doi.org/10.1007/ s11240-018-1433-6.

Sornkanok Vimolmangkang

Sornkanok.v@pharm.chula.ac.th

1 Department of Pharmacognosy and Pharmaceutical Botany, Faculty of Pharmaceutical Sciences, Chulalongkorn

University, Bangkok 10330, Thailand

2 Department of Biochemistry and Microbiology, Faculty of Pharmaceutical Sciences, Chulalongkorn University, Bangkok 10330, Thailand

3 Research Unit for Natural Product Biotechnology, Faculty of Pharmaceutical Sciences, Chulalongkorn University, Bangkok 10330, Thailand 